\title{
LEAST-SQUARES REVERSE TIME MIGRATION (LSRTM) IN THE SHOT DOMAIN
}

\author{
Antonio Edson Lima de Oliveira ${ }^{1}$, Reynam da Cruz Pestana ${ }^{2}$ and Adriano Wagner Gomes dos Santos ${ }^{3}$
}

\begin{abstract}
One of the major limitations of imaging methods is, usually, the incomplete recorded seismic data that cause difficulties for the subsurface imaging techniques. The least-squares migration method (LSM) was proposed in order to reduce migration artifacts caused by incomplete data. The LSM technique has been shown to be an efficient tool to provide sharp images, especially in critical areas, such as those affected by salt tectonics. However, the complete solution of LSM method requires the computing and storage of the Hessian matrix, which causes a very low computational efficiency. In order to overcome such challenges, LSM method was tested with reverse time migration (RTM) algorithm as the adjoint of modeling operator. We implemented two iterative methods where the migrated images are updated until an arbitrary number of iterations is reached. As a result, we obtained images with a reduction in the error between the modeled and calculated data. The least-squares reverse time migration (LSRTM) sections showed a better resolution than those obtained with the conventional RTM method.
\end{abstract}

Keywords: seismic imaging, resolution, modeling, iterative method, computational coast.

RESUMO. Uma das limitações das técnicas de imageamento é que, via de regra, os dados sísmicos registrados são incompletos. Isso impossibilita uma correta reconstituição dos refletores em subsuperfície. 0 método de migração por mínimos quadrados (LSM) foi proposto com o objetivo de reduzir os artefatos de migração provenientes de dados com uma amostragem irregular. A técnica LSM mostra-se eficiente como ferramenta na obtenção de imagens nítidas, especialmente em áreas tipicamente críticas para o imageamento, a exemplo daquelas afetadas por tectônica salífera. Em contrapartida, por conta da necessidade de cálculo e armazenamento da matriz Hessiana da solução LSM, a eficiência computacional do método é baixa. Com o objetivo de contornar tais dificuldades, implementou-se neste trabalho 0 método LSM tendo como adjunto do operador de modelagem o algoritmo reverso no tempo (RTM). Implementou-se dois métodos iterativos, nos quais as imagens migradas são atualizadas até um número arbitrário de iterações. Como resultado, obteve-se imagens com redução do erro entre os dados modelados e 0 de entrada para diferentes números de iterações. As seções LSRTM (Least-squares reverse time migration) obtidas mostram uma resolução melhor do que aquelas obtidas com 0 método RTM convencional.

Palavras-chave: imageamento sísmico, resolução, modelagem, método iterativo, custo computacional.

\footnotetext{
1 Petrobras, Avenida Antonio Carlos Magalhães, 1113, Torre Pituba, 20 andar, 41825-903 Salvador, BA, Brazil. Phone: +55(71) 3348-4182

- E-mail: aelol@yahoo.com.br

2Universidade Federal da Bahia, Instituto de Geociências, Centro de Pesquisa em Geofísica e Geologia, Campus Universitário da Federação, 40210-730 Salvador, BA, Brazil. Phone: +55(71) 3283-8512 - E-mail: pestana@cpgg.ufba.br

3 Instituto Nacional de Ciência e Tecnologia - Geofísica do Petróleo / Universidade Federal da Bahia - UFBA, Instituto de Geociências, Centro de Pesquisa em Geofísica e Geologia, Campus Universitário da Federação, 40210-730 Salvador, BA, Brazil. Phone: +55(71) 3283-8551 - E-mail: adrianowgs@gmail.com
} 


\section{INTRODUCTION}

Sharp and reliable images of the subsurface still remain a challenge, especially in high geological complexity areas. Some of these include inconsistency of the modeling equations, irregular sampling and computational requirements. The least-squares migration method was proposed in order to reduce migration artifacts arising from incomplete data (Nemeth et al., 1999). The difficulty is that the complete solution of LSM method requires the computing and storage of the Hessian matrix, generating a very low computational efficiency. Efforts have been done in order to find alternatives to their solution, and to minimized the computational cost associated with LSM method. Chen \& Wang (2010) used a compensation illumination term in reverse time migration (RTM) in the plane wave domain, which can be regarded as an approximation of the Hessian matrix. Huang et al. (2011) treated the image problem as a least-squares inversion problem, and the LSM algorithm that used was associated with the pre-stack depth Kirchhoff method. Dai et al. (2012) implemented LSM method with the standard RTM operator, compounding the least-squares reverse time migration (LSRTM) method. Dai \& Schuster (2013) presented a formulation of LSRTM method in the plane wave domain with the goal of reducing the amount of data and improving the computational efficiency.

In this paper, we implemented a solution similar to the one presented by Dai \& Schuster (2013), where the input data in the migration workflow was modeled from Born's operator with the smoothed velocity field and the reflectivity model. We implemented two LSRTM alternatives to update the results, where the migrated images are updated to an arbitrary number of iterations. The tests were performed on the synthetic dataset of the Marmousi model. After a few iterations, the imaging using LSRTM method showed better resolution when comparing with conventional RTM method.

\section{THEORY}

The inversion theory deals with the reverse problem. Thus, from the processing of a data set - and in most case with the introduction of some a priori information - we seek information about the statistics and the values of the model parameters. The goal of geophysical inversion is to solve problems like:

$$
d=g(M),
$$

where $d$ is the observed data, $M$ is the unknown model and $g$ is the forward modeling function.

Equation (1) describes non-linear problems, representing the majority of geophysical problems. However, locally, they can be transformed or approximate for linear versions, that can be described by the following relationship:

$$
\mathbf{d}=\mathbf{G m}
$$

where $\mathbf{d}=\left(d_{1}, d_{2}, \ldots, d_{M}\right)^{T}$ is the observation vector, $\mathbf{m}=\left(m_{1}, m_{2}, \ldots, m_{N}\right)^{T}$ is the parameters vector and $\mathbf{G}$ is the matrix that relates the space model to the data space. The correspondent inverse problem is given by:

$$
\mathbf{m}=\mathbf{G}^{-\mathbf{1}} \mathbf{d},
$$

in which $\mathbf{G}^{-\mathbf{1}}$ is the classical inverse.

The existence of the inverse of $\mathbf{G}$ in the Eq. (3) would provide the exact solution for the model $\mathbf{m}$, but is not feasible in geophysical problems. A common way to solve this problem is to use the least-squares method that provides an estimated model $\left(\mathbf{m}^{\text {est }}\right)$, which is best approximated to the true model $\left(\mathbf{m}^{\text {true }}\right)$, as the following expression:

$$
\mathbf{m}^{e s t}=\left[\mathbf{G}^{T} \mathbf{G}\right]^{-1} \mathbf{G}^{T} \mathbf{d},
$$

where $\mathbf{G}^{T}$ is an adjoint of the modeling operator $\mathbf{G}$. The estimated solution presented in Eq. (4) is named least-squares migration (LSM).

According to Clearbout (1992), a standard seismic migration operator can be considered as the adjoint of a seismic forward modeling operator. In other words, the seismic migration image problem $\mathbf{G}$ and $\mathbf{G}^{T}$ represent the modeling and migration operators, respectively. In this case, considering $\left[\mathbf{G}^{T} \mathbf{G}\right]^{-1} \approx \mathbf{I}$ in Eq. (4), we have the standard migration equation. i.e.:

$$
\mathbf{m}_{r}=\mathbf{G}^{T} \mathbf{d} .
$$

Equation (5) is the matrix equation for the standard migration method (RTM or Kirchhoff, for instance), where $\mathbf{G}^{T}$ represent migration operator and $\mathbf{m}_{r}$ is its result. The comparing between the results of LSM method (Eq. 4) with the standard method (Eq. 5) suggests that LSM solution provides better resolution images. The problem, however, is that $\mathbf{G}^{T} \mathbf{G}$ is the Hessian matrix, and its calculation has a high computational cost and very low efficiency. Thus, the LSM technique is presented in geophysical literature with different parameterizations. In this work we used an iterative solution similar to that one presented by Dai \& Schuster (2013), which is shown in the Appendix A.

\section{METHODOLOGY}

The processing workflow that was implemented in this work is illustrated in Figure 1. 


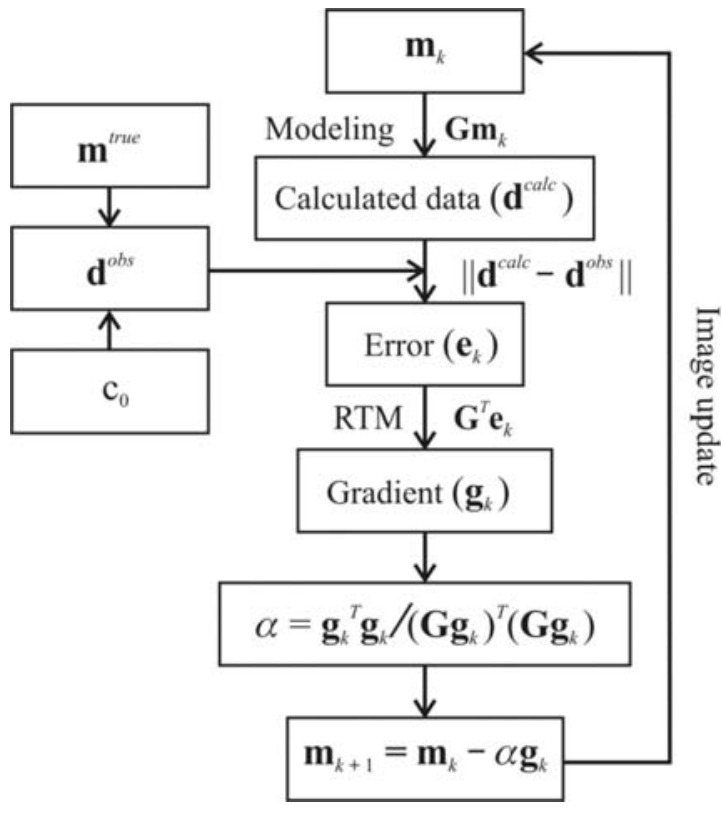

Figure 1 - LSRTM method processing workflow.

The reflectivity model $\left(\mathbf{m}^{\text {true }}\right)$ and the smoothed velocity field $\left(\mathbf{c}_{0}\right)$ from Marmousi model are inputs to computing the synthetic data using Born's modelling operator. The main parameters of Marmousi model are shown in Table 1. The velocity field grid number is 375 and 369 points for the vertical $(z)$ and horizontal $(x)$ directions, respectively. It was smoothed with 12 points in $z(n z=12)$ and 8 in $x(n x=8)$.

Table 1 - Main parameters of Marmousi model.

\begin{tabular}{|l|r|}
\hline Receiver interval $(\mathrm{m})$ & 25 \\
Source interval $(\mathrm{m})$ & 25 \\
Minimum offset $(\mathrm{m})$ & 200 \\
Maximum offset $(\mathrm{m})$ & 2575 \\
Sample interval (ms) & 2 \\
Record time (s) & 3 \\
Number of shots & 240 \\
Number of channels & 96 \\
\hline
\end{tabular}

The synthetic data generated in this process is considered as the observed data $\left(d^{o b s}\right)$ in Eq. (6). The next step is the chosen of the initial model or a priori model $\left(m_{0}\right)$, used to compute the calculated data $\left(d^{\text {calc }}\right)$. In this work we did not supply the initial model, then the error at the first iteration was the observed data itself. The residual error $\left(e_{k}\right)$ is the difference between the calculated and observed data, i.e.,

$$
\mathbf{e}_{k}=\left[\mathbf{G m}_{k}-\mathbf{G m}^{\text {true }}\right]=\left[\mathbf{d}^{\text {calc }}-\mathbf{d}^{\text {obs }}\right] .
$$

The error in the Eq. (6) is updated at each iteration. The next step is to obtain the model to the $k+1$ iteration. In the iterative solution presented by Nemeth et al. (1999), the migration operator $\mathbf{G}^{T}$ is applied to residual error to provide the migrated image correction $\mathbf{g}_{k}$, so that,

$$
\mathbf{g}_{k}=\mathbf{G}^{T} \mathbf{e}_{k} .
$$

Here we applied the RTM operator to compute the gradient $\left(\mathbf{g}_{k}\right)$. The model for the iteration $k+1$ is given by the following equation,

$$
\mathbf{m}_{k+1}=\mathbf{m}_{k}-\alpha \mathbf{g}_{k},
$$

where the parameter $a$ is the iteration step length shown in Eq. (9):

$$
\alpha=\frac{\mathbf{g}_{k}^{T} \mathbf{g}_{k}}{\left[\mathbf{G g}_{k}\right]^{T}\left[\mathbf{G g}_{k}\right]} .
$$

The step length $\alpha$ in Eq. (9) is calculated analytically at each iteration.

At the final step of each iteration, the current model $\mathbf{m}_{k}$ is updated by that one from iteration $k+1$. The final image is obtained by repeating the process until the desired error or the pre-set iterations number is reached. A detailed derivation of the least-squares reverse time migration is shown in the Appendix A.

To summarize, the main steps of the LSRTM method implemented in this work, which are illustrated in the flowchart of the Figure 1 are:

1. Computing the observed data from the smoothed velocity field and the reflectivity model using Born's modelling operator;

2. Initial model;

3. Modeling of the calculated data from the initial model;

4. Computing of the residual error between calculated and observed data;

5. Migration of the error employing the RTM operator;

6. Gradient calculation;

7. Step length calculation for each iteration;

8. Current model update; and

9. Repeating the process for an arbitrary number of iterations.

We implemented two iterative methods. First being the image update method, where the image acquired at the end of the current iteration was the input to the following one. In this case, the update process is repeated for a number of iterations previously set so that the image obtained in the $k$-th iteration is the final section, as shown in Figure 2. 


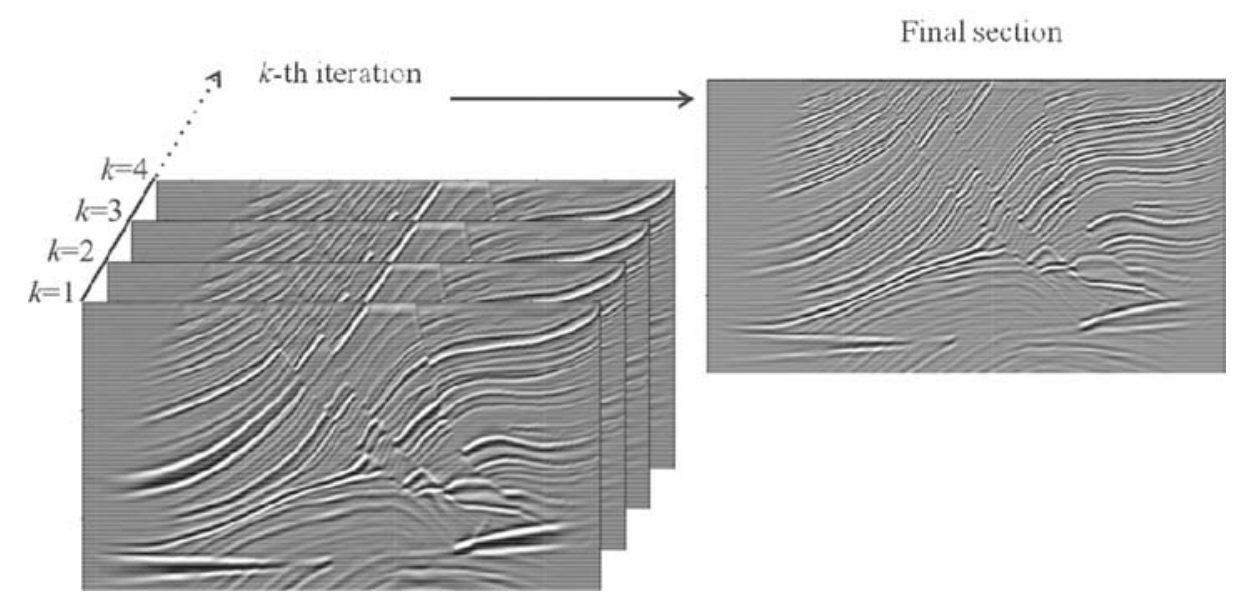

Figure 2 - Schematic flow of the image update LSRTM method.

The second method is the migrated shot image update method, in which each image migrated of all shots is updated to an arbitrary number of iterations is reached. The images migrated of the $i$-th shots obtained at $k$-th iteration are added to compose the final section, as shown in Figure 3. In this process, the shot image achieved at current iteration is the input to the next one. In both methods, the processing flow is the same illustrated in Figure 1 and previously enumerated. The same workflow was applied to both methods.

At each iteration of LSRTM method, two modeling and one migration is required. The first modeling is performed from the current model in order to provide the calculated data and the second modeling is done from the gradient vector providing the iteration step length $\alpha$. The RTM operator is applied at residual error providing the gradient vector. This is one of the features responsible for the high computational cost of the method.

There are several criteria to stop iterative process. In this work we have set a fixed number of iterations. Thus, the process will be finished upon reaching the number of iterations previously set.

\section{RESULTS}

The 2D Marmousi model was used to test the efficiency LSRTM method implemented. First it was migrated with standard prestack RTM method in the common shot gather domain. The input data and the smoothed velocity field was the same used in the LSRTM workflow. The result is shown in Figure 4.

The image update LSRTM method was first tested with incomplete data. Thus, the input data in the processing workflow,

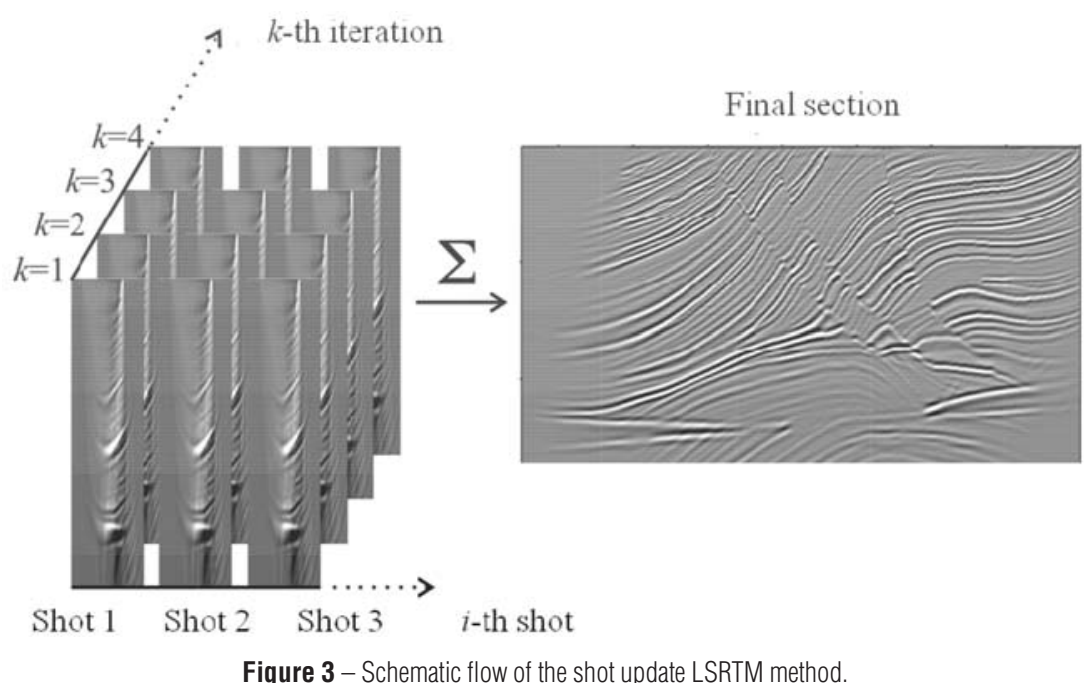




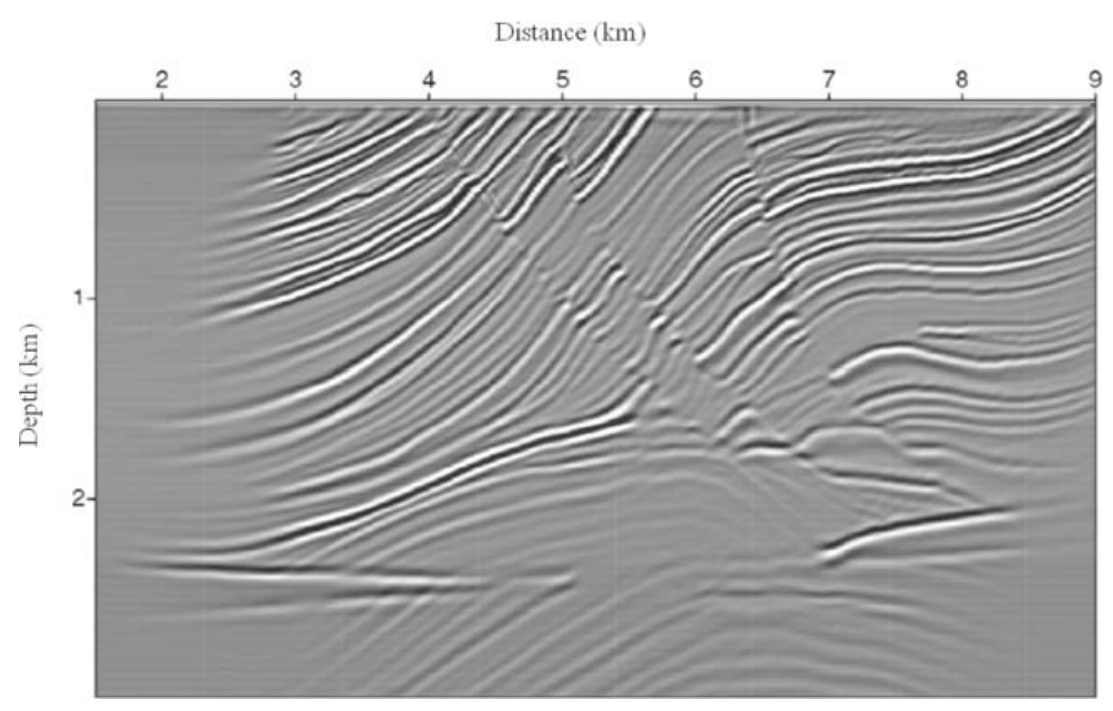

Figure 4 - Conventional pre-stack RTM section in the shot domain.

originally composed of 240 shots, was reduced to 15 shots equidistant of $400 \mathrm{~m}$. The objective of this test was to verify the efficiency of the method when applied over incomplete data. Figure 5 shows the resulted image after 30 iterations. Although the data was incomplete, the image resulting is relatively clear.

After that the method was tested with complete data, which is composed of 240 shots $25 \mathrm{~m}$ spaced. Figures 6 and 7 are the results after 1 and 30 iterations, respectively.

These figures show that the image update LSRTM method provides sharper images within the first iteration. The images obtained from the incomplete data show good continuity of reflectors, especially those that are deeper. With the complete data, image sharpness is good throughout the entire section after the first iteration. The reflectors are continuous and the faults are well defined.

The residual error is the parameter used to evaluate if the method tends towards the model that minimizes the difference between input and calculated data along the iterative process. Figure 8 shows its evolution as a function of increasing the number of iterations. The graph indicates the trend error for images obtained from complete and incomplete data, from the iteration 1 to 45 . In both cases, the error decreases quickly from early iterations and from 30 iterations there is no reduction in the error that justifies the maintenance of the iterative process. However, based

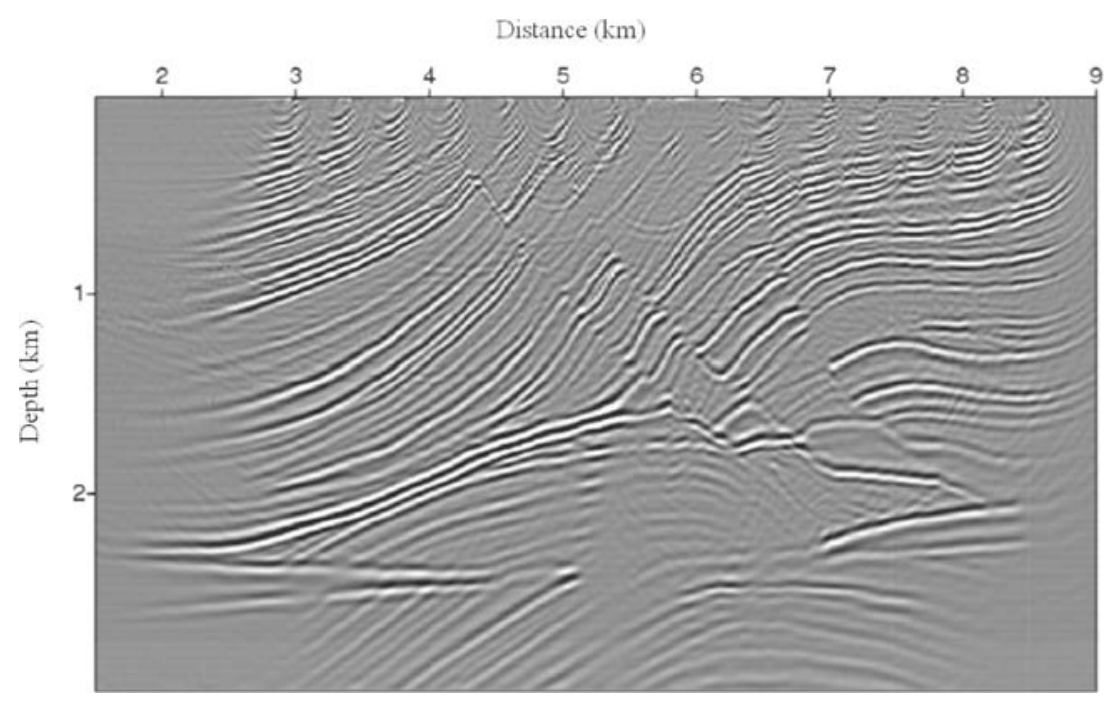

Figure $\mathbf{5}$ - Image update LSRTM section: 15 shots after 30 iterations. 


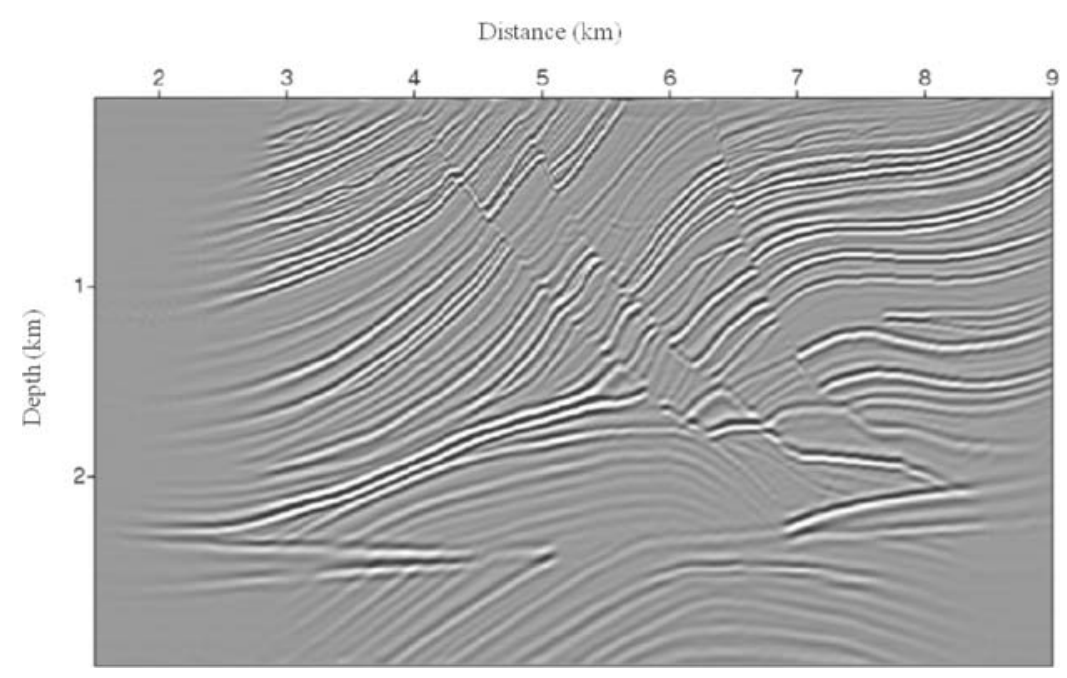

Figure 6 - Image update LSRTM section: 240 shots after 1 iteration.

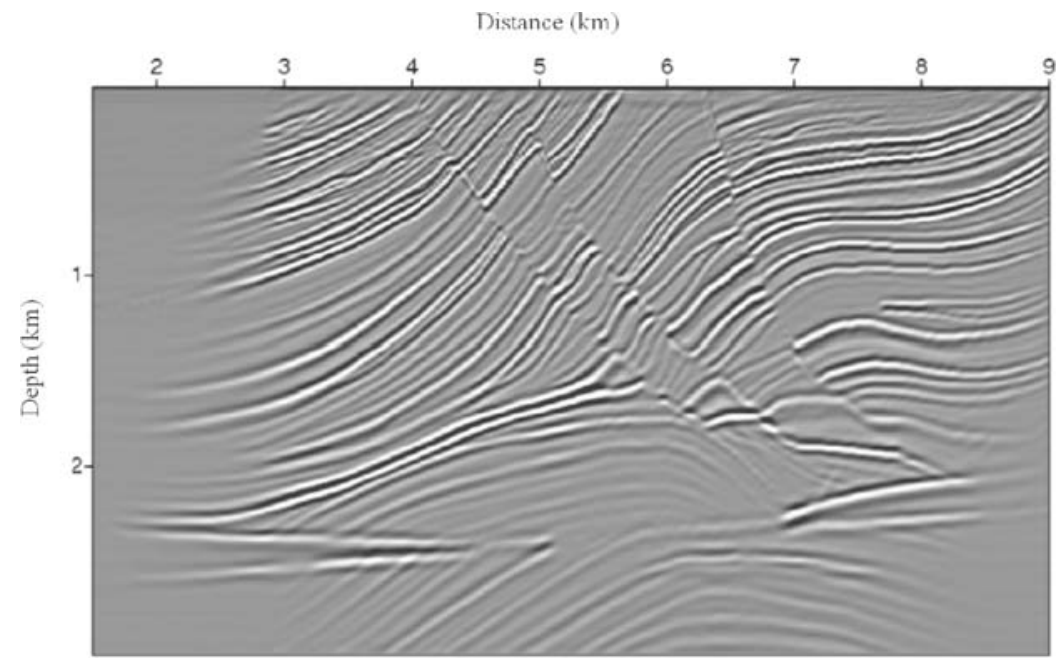

Figure 7 - Image update LSRTM section: 240 shots after 30 iterations.

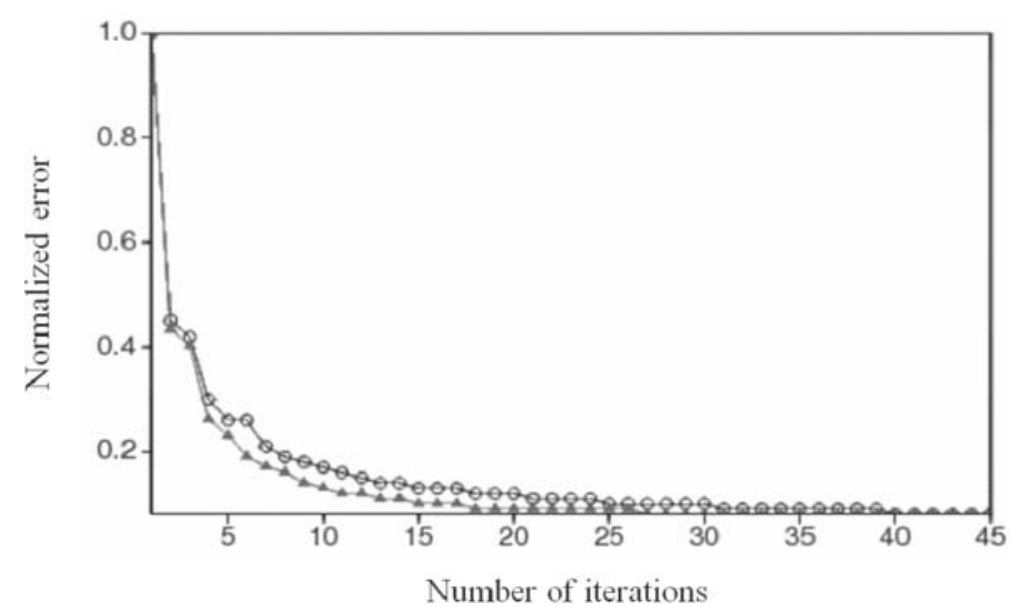

Figure 8 - Residual error versus the iteration number: 15 shots (circles) and 240 shots (triangles). 


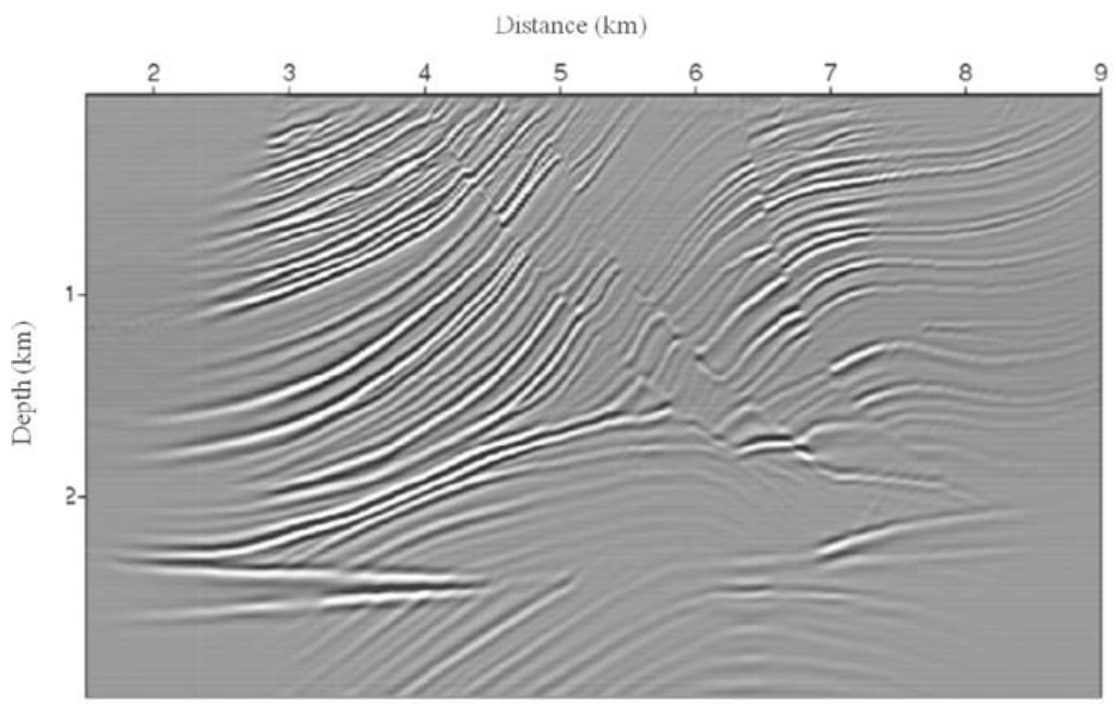

Figure 9 - Shot update LSRTM section: 240 shots after 1 iteration.

on a simple qualitative evaluation, the process could be stopped from the early iterations - fifth for example - without significant image quality losses.

The test with the shot update LSRTM method was initially performed with the original data (240 shots). The Figure 9 shows its result after 1 iteration. In this image, the faults and reflectors are well delineated. However, when compared with those obtained from the image update LSRTM method, the image sharpness is lower, especially in the central part of the model, where higher angle reflectors are present.

One of the reasons why the LSM method has been widely studied recently is its efficiency when applied to incomplete data. LSM process helps reduce artifacts in a natural way by generating the model that predicts the observed data in a least-squares sense (Nemeth et al., 1999). Thus, we excluded 30 shots of the Marmousi model, by simulating a $775 \mathrm{~m}$ gap. This data was migrated with both LSRTM methods and with standard RTM algorithm. The resultant sections are shown from Figures 10 to 12 .

In these images, there is a shadow zone that affects especially the shallower part of the sections, between the horizontal distances from $5.5 \mathrm{~km}$ to $6.5 \mathrm{~km}$, which is the range of the gap, and until approximately $1 \mathrm{~km}$ of depth. Although it spreads

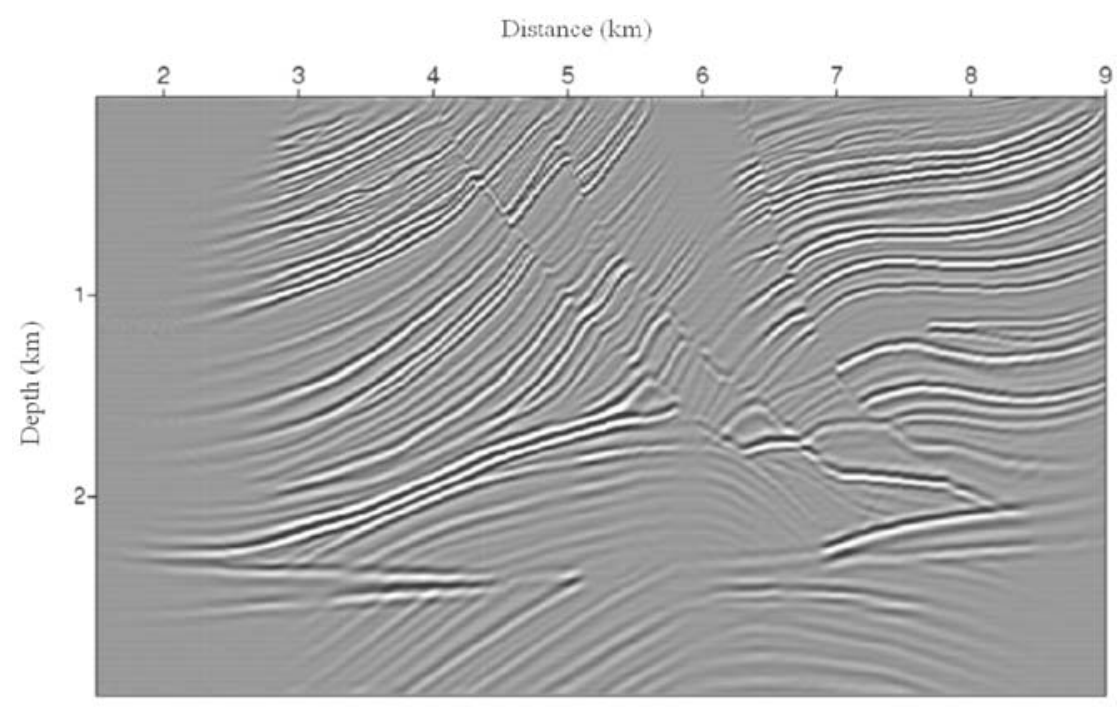

Figure 10 - Image update LSRTM section after 30 iterations. Data with 30 shots gap. 


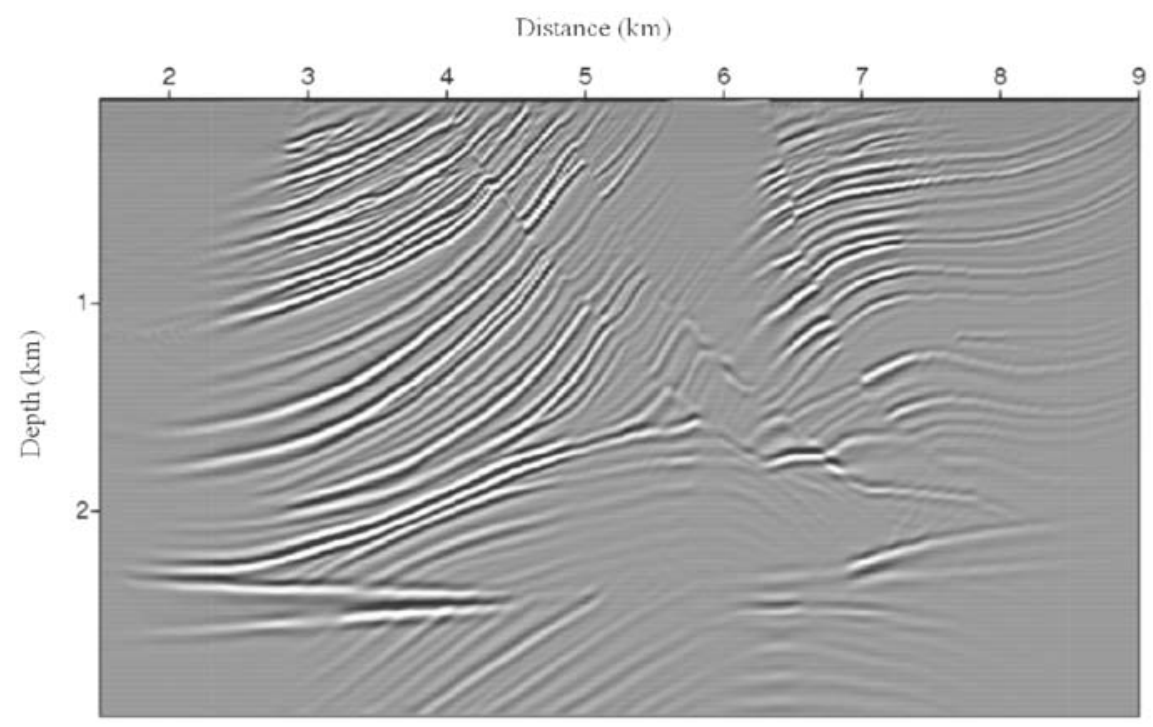

Figure 11 - Shot update LSRTM section after 30 iterations. Data with 30 shots gap.

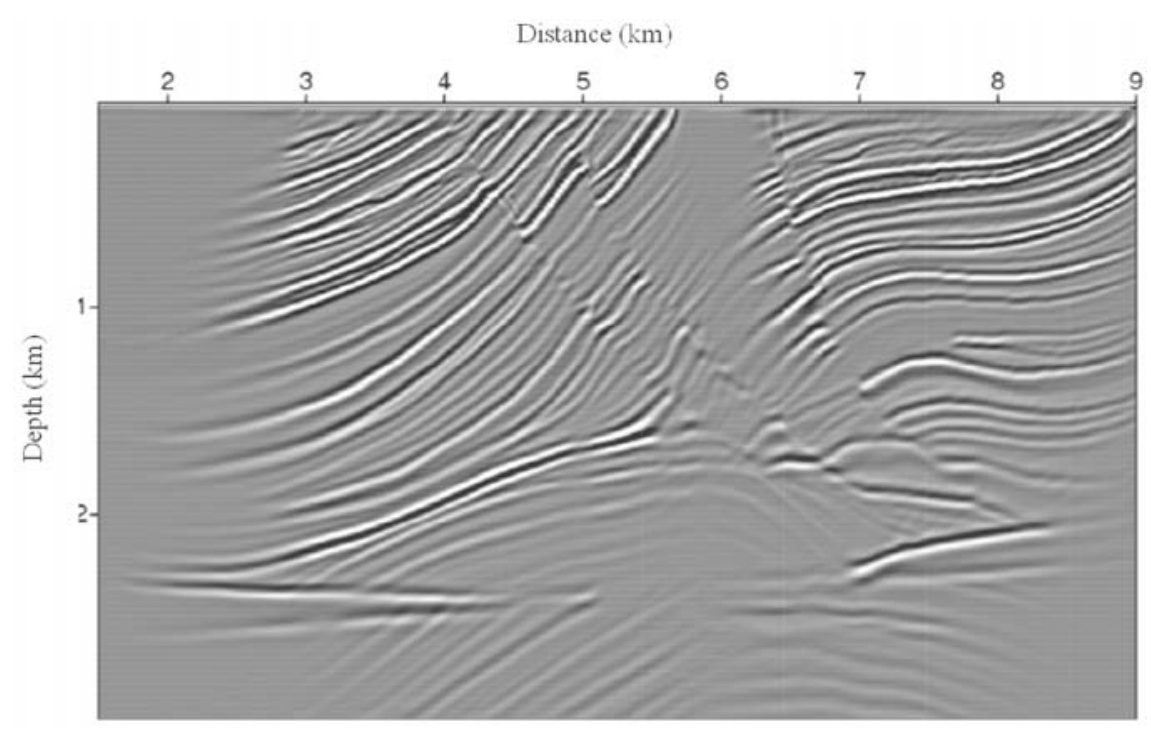

Figure 12 - Conventional RTM section. Data with 30 shots gap.

throughout the section, its effect is attenuated with the depth. This result confirms the efficiency of the method when applied to incomplete data. Comparing with the conventional RTM result, one realizes that the resolution of LSRTM images are better. In the gap area, the imaging of reflectors is more efficient, especially in the deepest parts of the model.

The processing time is one of the critical factors for the imaging techniques. In order to get information about the computational cost, we performed the test of processing time using a cluster with 120 units with the same performance. Figure 13 shows a chart, in decimal time, with computing time that was re- quired by conventional RTM method and LSRTM method after 1 and 30 iterations. It is clear that the LSRTM computational cost is high when compared with the RTM computational cost. The time processing required for the RTM method was approximately 7 minutes, amount which corresponds to approximately $30 \%$ of that one spent on a single iteration of LSRTM method.

\section{CONCLUSIONS}

The tests performed on synthetic data form 2D Marmousi model with image update LSRTM method and migrated shot image update LSRTM method provided sharp images from first iteration. 


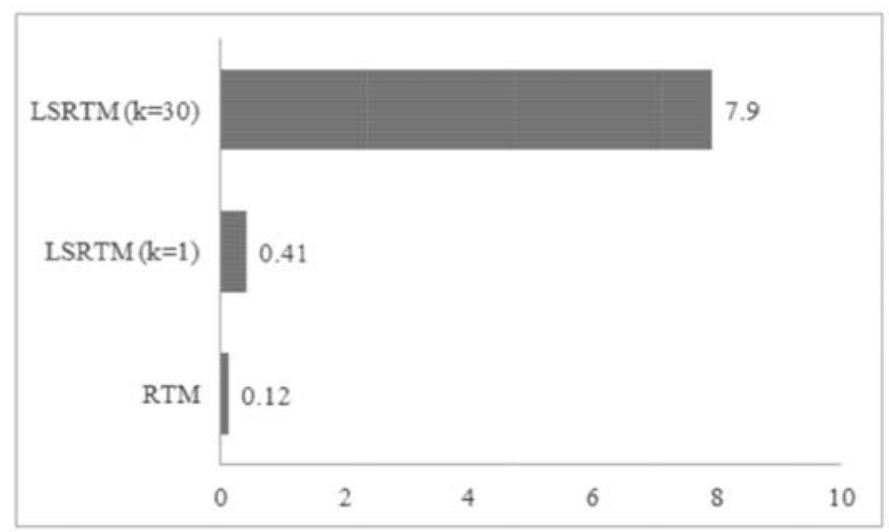

Decimal time

Figure 13 - Processing time: conventional RTM and LSRTM after 1 and 30 iterations.

In this image, the faults and reflectors are well delineated. The differences between the reflectors from the two LSRTM methods implemented are small. However, those obtained with the image update LSRTM processing flow are qualitatively better. The sections from incomplete data show good reflectors continuity, especially from the deepest ones. This result confirms the efficiency of the method when applied to data with acquisition gaps.

The residual error decreases quickly from early iterations. The trend shows that from 30th iteration there is no reduction in its value that justifies the maintenance of the iterative process. However, based on a simple qualitative evaluation, the process could be stopped from the early iterations, without significant image quality losses.

The computational processing cost of LSRTM method is high compared with the RTM method. The processing time required for the RTM was about $30 \%$ of that used to run a single LSRTM iteration. Therefore, the main challenge for the use of the LSRTM method in large scale is its computational cost.

Despite the outlined problems, the LSRTM method appears to be quite promising as a seismic imaging tool. It has attracted a growing interest in the geophysics field, which explains the large number of technical papers that presented least-squares migration with different implementations in early years. Most of these papers presented results or provide some alternatives to increase the performance of the method. Some examples are: (1) implementation of graphics processing unit (GPU) version; the use of data in the ray parameters domain or (3) the use of optimization methods that accelerate the iterative search process.

Finally, the LSM method can be thought of as an auxiliary tool, which can be applied at the imaging of the specific areas where doubts remain after the imaging with conventional tools.
It can still be used in areas where old data exists, and new acquisitions face restrictions such as the environmental permit process, urban occupation, infrastructure works and oil production facilities among others.

\section{ACKNOWLEDGEMENTS}

The authors thank Petrobras for the opportunity offered to perform this research in the form of a master's degree in Geophysics and to the LAGEP-CPGG-UFBA for the resources available during its realization.

\section{APPENDIX A}

\section{Least-squares reverse time migration}

In this paper, we implemented a solution similar to the one presented by Dai \& Schuster (2013), where the input data in the migration workflow was modeled from Born's operator with the smoothed velocity field and the reflectivity model. Thus, assuming the constant density acoustic wave equation

$$
\frac{1}{c_{0}} \frac{\partial^{2} p_{0}}{\partial t^{2}}-\nabla^{2} p_{0}-s=0
$$

where $c_{0}=c_{0}(\mathbf{x})$ is a background velocity model, and $p_{0}=p_{0}\left(\mathbf{x}, t ; \mathbf{x}_{s}\right)$ is the pressure field associated with the source term $s=s(\mathbf{x}, t)$. A perturbation in the velocity model $c=c_{0}+\delta c$ produce a wavefield

$$
p=p_{0}+\delta p,
$$

which satisfy the following acoustic-wave equation

$$
\frac{1}{c^{2}} \frac{\partial^{2} p}{\partial t^{2}}-\nabla^{2} p=s
$$




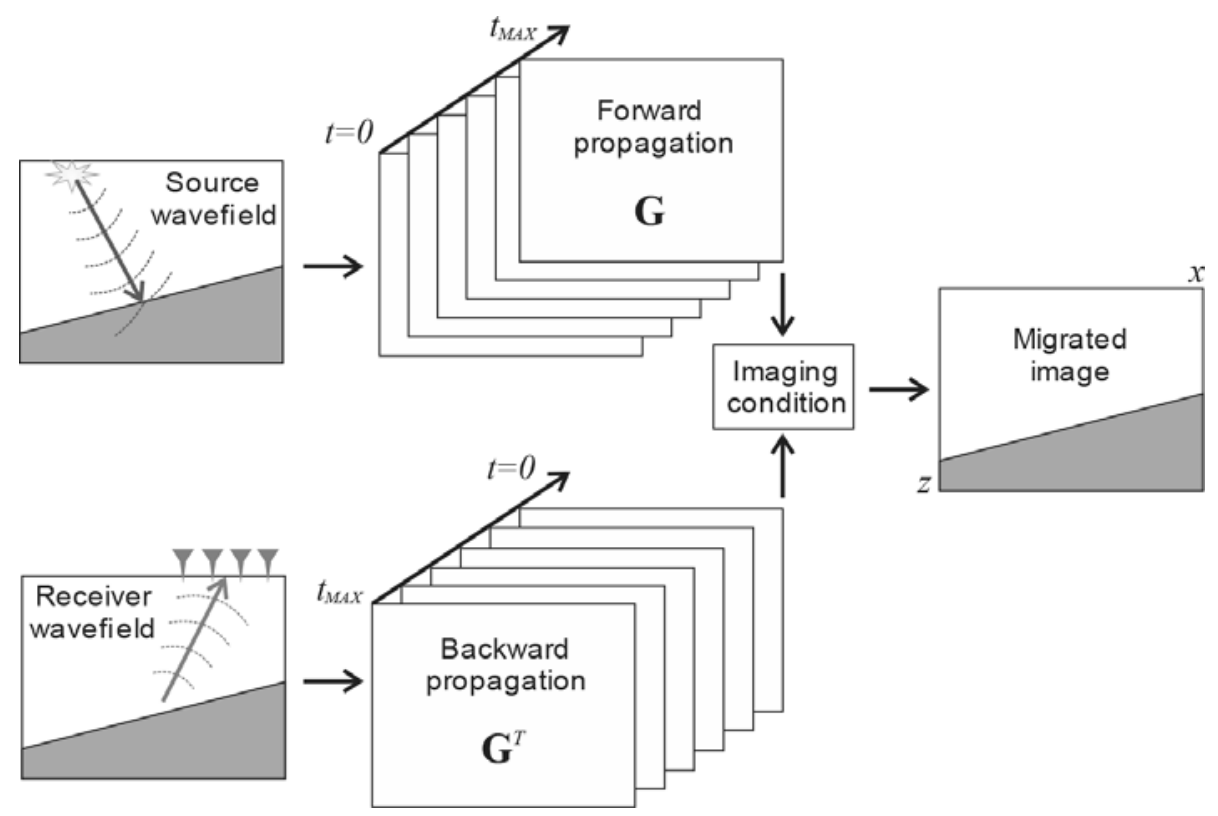

Figure A1 - Source and receiver wavefields propagation scheme.

The velocity term can be expanded as follows

$$
\frac{1}{\left(c_{0}+\delta c\right)^{2}} \approx \frac{1}{c_{0}^{2}}-\frac{2 \delta c}{c_{0}^{3}} .
$$

Subtracting Eq. (A2) from Eq. (A4) and using Eq. (A1), yields the wave equation for the wavefield perturbation $\delta p$ :

$$
\frac{1}{c_{0}^{2}} \frac{\partial^{2} \delta p}{\partial t^{2}}-\nabla^{2} \delta p=\frac{\partial^{2} p}{\partial t^{2}} \frac{2 \delta c}{c_{0}^{3}}+O\left(c^{2}\right) .
$$

Applying the Born approximation to the right-hand side $p \approx p_{0}$, neglecting the higher-order terms $O\left(c^{2}\right)$ and defining the reflectivity model as $m=2 \delta c / c_{0}$, Eq. (A5) becomes

$$
\frac{1}{c_{0}^{2}} \frac{\partial^{2} \delta p}{\partial t^{2}}-\nabla^{2} \delta p=m \frac{1}{c_{0}^{2}} \frac{\partial^{2} p_{0}}{\partial t^{2}}
$$

where $m=m(\mathbf{x})$.

The calculation of the reflection data $\delta p$ requires two finitedifference extrapolations in order to solve Eq. (A1) to provide the wavefield $p_{0}$ and one to solve Eq. (A6) for the reflection data $\delta p$. The migration operation of a shot gather $d=d\left(\mathbf{x}_{g}, t ; \mathbf{x}_{s}\right)$, representing the wavefield recorded at the receiver position $\mathbf{x}_{g}$, requires two finite-difference extrapolation, as shown in Eq. (A7) and Eq. (A8):

$$
\frac{1}{c_{0}^{2}} \frac{\partial^{2} p_{0}}{\partial t^{2}}-\nabla^{2} p_{0}=s
$$

and,

$$
\frac{1}{c_{0}^{2}} \frac{\partial^{2} p}{\partial t^{2}}-\nabla^{2} q=d
$$

The source wavefield $p_{0}$ propagates forward in time while the receiver wavefield $q$ propagates backward in time, as shown by Figure $\mathrm{A} 1$ including the imaging condition.

The migrated image associated with the shot at $\mathbf{x}_{s}$ is generated by applying the imaging condition

$$
m=\sum_{t} \frac{1}{c_{0}^{2}} \frac{\partial^{2} p_{0}}{\partial t^{2}} \cdot q .
$$

In the context of least-squares migration, the solution to Eq. (A6) can be represent as the following matrix operation

$$
\mathbf{d}_{i}=\mathbf{G}_{i} \mathbf{m},
$$

where $\mathbf{d}_{i}$ represent the reflection data vector for the $i$ th shot, $\mathbf{m}$ represents the migration image which in this case is related to the perturbation in velocity, and $\mathbf{G}_{i}$ represents the Born modeling operator related with the ith shot. In the same way, the RTM operator can be written

$$
\mathbf{m}_{m i g, i}=\mathbf{G}_{i}^{T} \mathbf{d}_{i},
$$

where $\mathbf{m}_{m i g, i}$ represent the migration image for the $i$ th shot and $\mathbf{G}_{i}^{T}$ represent the migration operator associated with the $i$ th shot. Thus, for a dataset with $\mathbf{N}_{s}$ shots, the final image is the stack of migrated images from all of the individual shots, as shown in Eq. (A12):

$$
\mathbf{m}_{m i g}=\sum_{i=1}^{N_{s}} \mathbf{G}_{i}^{T} \mathbf{d}_{i} .
$$




\section{REFERENCES}

CHEN SC \& WANG HC. 2010. Migration compensation with plane wave illumination. Chinese Journal of Geophysics, 53(4): 583-589.

CLEARBOUT JF. 1992. Earth soundings analysis: Processing versus Inversion. Blackwell Scientific Publications, Inc., Stanford-USA, 316 pp.

DAI W \& SCHUSTER GT. 2013. Plane-wave Least-squares Reverse Time Migration. Geophysics, 78: S165-S177.
DAI W, FOWLER P \& SCHUSTER GT. 2012. Multi-source least-squares reverse time migration. Geophysical Prospecting, 60: 681-695.

HUANG J, LI Z \& LIU Y. 2011. The least square pre-stack depth migration method on complex media. In: SPG/SEG Shenzhen 2011 International Geophysical Conference Technical Program Expanded Abstracts, 2263-2271.

NEMETH T, CHENGJUN W \& SCHUSTER GT. 1999. Least-squares migration of incomplete reflection data. Geophysics, 64(1): 208-221.

Recebido em 1 abril, 2015 / Aceito em 21 outubro, 2016

Received on April 1, 2015 / Accepted on October 21, 2016 